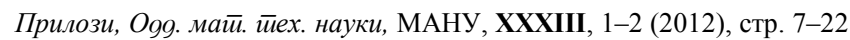
Contributions, Sec. Math. Tech. Sci., MANU, XXXIII, 1-2 (2012), pp. 7-22

ISSN 0351-3246

UDC: $519.216: 519.179 .2$

\title{
TOPOLOGY INDEPENDENT SIRS PROCESS
}

\author{
Igor Tomovski, Igor Trpevski, Ljupčo Kocarev
}

\begin{abstract}
A b s t r a c t: Following our recent work on the topology independent SIS spreading model $[25,26]$, in this article we analyze a SIRS type of a spreading processes, taking place on complex networks characterized by a special form of contact dynamics, for which we use the term "acquisition exclusivity". We show that, identically as for the SIS type process, for the studied set-up, in circumstances where statistical independence of joint events may be assumed, analytical solution for the probabilities that each node is in a certain status in stationary regime may be found. Furthermore, the obtained results indicate that the SIRS process, under the analyzed circumstances is topology independent.
\end{abstract}

Key words: complex networks; SIRS process; nonlinear systems

\section{INTRODUCTION}

In the last decade, the theory of complex networks became one of the most exciting and fastest growing research areas in the modern science. This was primarily due to the rapid development of communication networks, especially the internet and the wireless communications. However, the serious growth of the transport, power distribution and other infrastructure networks, significantly contributed to this interest as well. A group of research problems most commonly studied on complex networks was the one related to network security, with the emphasis on stochastic spreading processes. Numerous studies treated different security problems, starting from processes of virus spreading and virus spreading control [1, 2, 3, 4, 5, 6, 7, 8, 9, 10, 11, 12, 14, 15], cas- 
cading failures and blackouts $[16,17,18,19,20,21]$, attack vulnerabilities [22, $23,24]$ etc.

When investigating the stochastic spreading processes on networks, most authors have addressed the issue from the aspect of their potential threat on the networks integrity and functionality. Lately, the social networks have invoked an interest in these processes from the aspect of their role in information exchange and social interactions. In that context, in our recent work [25, 26], we have studied their potential use for controlled spreading of content on technological networks.

In $[25,26]$, we investigated a spreading process of SIS type taking place on networks, characterized by a special form of contact dynamics, for which the term "acquisition exclusivity" was used. As explained in these papers, we use the term "acquisition exclusivity" to denote a network contact behavior where at each instance every node in the network randomly contacts and acquires material from exactly one of its neighbors, with a certain probability. Spreading processes where similar types of "non-regular" contact/spreading behavior occurs, for various reasons, had also been analyzed in the literature, for example the contact process [27, 28], the Voter model [29] etc. Adopting the approach of Wang et al. [6] and Chakrabarti et al. [7], assuming that the hypothesis of statistical independence of joint events holds we showed that the SIS process under the studied circumstances is analytically solvable and topology independent. Numerical investigations indicated that the validity of these results was dependent on the region where the process was analyzed, primarily due the validity of the hypothesis of statistical independence of joint events. The analysis showed that the obtained analytical results are valid for networks with large minimal node degree, for high $\beta / \delta$ ratios and in circumstances where $\beta \rightarrow 1$. We argued that the importance of these results lays in their applicability for controlled spreading of useful content in technological networks. We suggested that applications for controlled spreading of content may be developed. These applications should form virtual networks using the infrastructure of existing communication networks (lans, Internet etc). Useful content is shared through the virtual networks as a process of SIS type, where the number (statistical expectation) of individuals (nodes) that posses the content at an instance in time is controlled by adequate combination of parameters $\beta$ and $\delta$.

In this paper we extend these results to the spreading processes of SIRS type. Again in the analysis we adopt the approach of Wang et al. [6] and Chakrabarti et al. [7] and assume that the statistical independence of joint events holds. We show that, under the circumstances, an analytical solutions for the 
probabilities that each node in the network is either susceptible, infected or recovered/removed, may be found. The analysis shows that, exactly as for the SIS process, the obtained results are topology independent. We analyze the validity of the obtained results by performing stochastic analyzes on synthetic (computer generated) networks and derive conclusions in respect to the parametric and topological regions where the analytical results truly mimic the reality.

\section{DESCRIPTION OF THE ANALYZED PROCESS}

We observe a SIRS type of a spreading process occurring on network which is topologically represented by a graph that is strongly connected, unweighed and undirected. The network functions in acquisition exclusivity mode of contact dynamics. Let $\mathbf{A}=\left[a_{i j}\right]$ be the graphs ' 0 ' - '1' adjacency matrix; due to the prerequisites related to the characteristics of the graph $\mathbf{A}$ is symmetric and irreducible. With $p(i \leftarrow j)$ we denote the contact probability, i.e. the probability that node $i$ will contact node $j$ at an instance of time and, possibly, acquire some form of spreading content. Due to acquisition exclusivity, $\sum_{j=1, N} p(i \leftarrow j)=1$. Next, the contact probability $p(i \leftarrow j)>0$ is strictly positive when $a_{i j}=1$, and in general the contact probabilities $p(i \leftarrow j) \neq p(j \leftarrow i)$. Therefore, the matrix $B=[b \mathrm{ij}]$, where $b_{i j}=p(i \leftarrow j) a_{i j}$ is irreducible and asymmetric, satisfying $\sum_{j=1, N} b_{i j}=1$. It is assumed that no instantaneous reinfection may occur within one time step for both type of processes.

In respect to the constrains given above, and using the approach of Wang et al. [6] and Chakrabarti et al. [7], the SIRS type process is described with the following set of probability equations:

$$
\begin{gathered}
p_{i}^{S}(t+1)=p_{i}^{S}(t)\left(1-\sum_{j=1}^{N} \beta b_{i j} p_{j}^{I}(t)\right)+\gamma p_{i}^{R}(t), \\
p_{i}^{I}(t+1)=p_{i}^{S}(t) \sum_{j=1}^{N} \beta b_{i j} p_{j}^{I}(t)+(1-\delta) p_{i}^{I}(t) \\
p_{i}^{R}(t+1)=\delta p_{i}^{I}(t)+(1-\gamma) p_{i}^{R}(t) \\
1=\delta p_{i}^{I}(t)+(1-\gamma) p_{i}^{R}(t)
\end{gathered}
$$

In (1), $p_{i}^{S}(t), p_{i}^{I}(t), p_{i}^{R}(t)$, define the probabilities that the node $i$ is in status susceptible, infected or removed, at time $t$, respectively. Parameter $\delta$ denotes 
the probability that an infected node will recover and $\gamma$ the probability that a removed node will become susceptible, both within one time step.

The following solutions satisfy (1):

- $\mathbf{p}^{I}=\mathbf{0}_{N \times 1}$, from which $\mathbf{p}^{R}=\mathbf{0}_{N \times 1}$ and $\mathbf{p}^{S}=\mathbf{1}_{N \times 1}$, with $\mathbf{0}$ and $\mathbf{1}$ being vectors whose elements are all zeros and ones, respectively.

- $\mathbf{p}^{S}=\left[p_{i}^{S}\right]$ such that $p_{i}^{S}=\frac{\delta}{\beta}$, for $i=\overline{1, N}$, yielding $\mathbf{p}^{I}=\left[p_{i}^{I}\right]$, where $p_{i}^{I}=\frac{1-\frac{\delta}{\beta}}{1+\frac{\delta}{\gamma}}$ and $\mathbf{p}^{R}=\left[p_{i}^{R}\right]$, such that $p_{i}^{R}=\frac{\delta}{\gamma} \frac{1-\frac{\delta}{\beta}}{1+\frac{\delta}{\gamma}}$ for all $i=\overline{1, N}$.

\section{DYNAMICAL ANALYSIS OF THE STUDIED SET-UP}

In this section a dynamical analysis of the system (1) is presented. The aim of the analysis is to confirm the dynamical stability of the fixed points obtained in the previous section.

Theorem 1. For the system described with the set of equations (1) and $\delta>\beta$, the fixed point that corresponds to the state described with the vector $\left[\left(\mathbf{p}^{\mathrm{S}}\right)^{T}\left(\mathbf{p}^{I}\right)^{T}\left(\mathbf{p}^{R}\right)^{T}\right]^{T}=\left[(\mathbf{1})^{T}(\mathbf{0})^{T}(\mathbf{0})^{T}\right]^{T}$ is globally asymptotically stable.

Proof. By rewriting the second equation of the system (1), in a vector form, one obtains:

$$
\begin{aligned}
\mathbf{p}^{I}(\mathrm{t}+1) & =((1-\delta) \mathbf{I}+\beta \mathbf{D}(t) \mathbf{B}) \mathbf{p}^{I}(t)=\mathbf{S}(\mathrm{t}) \mathbf{p}^{I}(t) \\
& =\mathbf{S}(t) \mathbf{S}(t-1) \ldots \mathbf{S}(0) \mathbf{p}^{I}(0)<\mathbf{S}^{t+1} \mathbf{p}^{I}(0),
\end{aligned}
$$

where $\mathbf{S}(t)=(1-\delta) \mathbf{I}+\beta \mathbf{D}(\mathrm{t}) \mathbf{B}$ and $\mathbf{S}=(1-\delta) \mathbf{I}+\beta \mathbf{B}$. Since $\lambda_{1 S}=1-\delta+\beta<1$ :

$$
\lim _{t \rightarrow \infty} \mathbf{S}^{t} \mathbf{p}^{I}(0)=\mathbf{0}
$$

Note that, exists $d>d i a$, such that for $t>d$ product of matrices $\mathbf{S}(t)$ is a strictly positive matrix (has strictly positive components). From that, the relation (2) and since $\mathbf{p}^{I}(0) \geq 0$, it may be concluded that for arbitrary $\mathbf{p}^{I}(0)$ : 


$$
\lim _{t \rightarrow \infty} \mathbf{p}^{I}(0)=\mathbf{0}
$$

From the third equation of the system (1):

$$
\lim _{t \rightarrow \infty} \mathbf{p}^{R}(t+1)=\lim _{t \rightarrow \infty}\left(\delta \mathbf{p}^{I}(t)+(1-\gamma) \mathbf{p}^{R}(t)\right)=(1-\gamma) \lim _{t \rightarrow \infty} \mathbf{p}^{R}(t),
$$

it is obvious that:

$$
\lim _{t \rightarrow \infty} \mathbf{p}^{R}(t)=\mathbf{0}
$$

Finally, from the normalization condition, the fourth equation of the system (1), one obtains that:

$$
\lim _{t \rightarrow \infty} \mathbf{p}^{S}(t)=\mathbf{1}
$$

The proof is completed.

Next, it will be shown that for $\beta>\delta$, the fixed point $p_{i}^{S}=p^{S}=\frac{\delta}{\beta}$, $p_{i}^{I}=p^{I}=\frac{1-\frac{\delta}{\beta}}{1+\frac{\delta}{\gamma}}$ and $p_{i}^{R}=p^{R}=\frac{\delta}{\gamma} \frac{1-\frac{\delta}{\beta}}{1+\frac{\delta}{\gamma}}$ for all $i=\overline{1, N}$ is valid solution for the system described with (1). In order to do so, it will be proven that the fixed point under observation is locally stable, while the other fixed point $p_{i}^{S}=p^{S}=1, p_{i}^{I}=p^{I}=0$ and $p_{i}^{R}=p^{R}=0, \quad i=\overline{1, N}$ is unstable.

Theorem 2. For the SIRS type spreading process defined with a set of the status probability equations (1) and $\frac{\delta}{\beta}<1$, the fixed point $p_{i}^{S}=p^{S}=1$, $p_{i}^{I}=p^{I}=0$ and $p_{i}^{R}=p^{R}=0, i=\overline{1, N}$, is locally unstable.

Proof. First, the system (1) will be rewritten in the following form:

$$
\begin{aligned}
& p_{i}^{S}(t+1)=1-p_{i}^{I}(t+1)-p_{i}^{R}(t-1) \\
& p_{i}^{I}(t+1)=\left(1-p_{i}^{I}(t)-p_{i}^{R}(t)\right) \sum_{i=1}^{N} \beta b_{i j} p_{j}^{I}(t)+(1-\delta) p_{i}^{I}(t) \\
& p_{i}^{R}(t+1)=\delta p_{i}^{I}(t)+(1-\gamma) p_{i}^{R}(t)
\end{aligned}
$$


By perturbing the second and the third equation of the system (3), at the observed point, one obtains:

$$
\begin{aligned}
& \delta p_{i}^{I}(t+1)=(1-\delta) \delta p_{i}^{I}(t)+\sum_{j=1}^{N} \beta b_{i j} \delta p_{j}^{I}(t) \\
& \delta p_{i}^{R}(t+1)=\delta\left(\delta p_{i}^{I}(t)\right)+(1-\gamma) \delta p_{i}^{R}(t)
\end{aligned}
$$

or in matrix form:

$$
\left[\begin{array}{l}
\delta \mathbf{p}^{I}(t+1) \\
\delta \mathbf{p}^{R}(t+1)
\end{array}\right]=\left[\begin{array}{cc}
(1-\delta) \mathbf{I}+\beta \mathbf{B} & 0 \\
\delta \mathbf{I} & (1-\gamma) \mathbf{I}
\end{array}\right]\left[\begin{array}{l}
\delta \mathbf{p}^{I}(t) \\
\delta \mathbf{p}^{R}(t)
\end{array}\right]=\mathbf{M}\left[\begin{array}{l}
\delta \mathbf{p}^{I}(t) \\
\delta \mathbf{p}^{R}(t)
\end{array}\right]
$$

In order to determine the local stability of the observed fixed point, analysis of the eigenvalues of the perturbation matrix $\mathbf{M}$ should be performed. As can be concluded from (5) the eigenvalues of $\mathbf{M}$ are $\lambda_{(1 \div N)_{M}}=\lambda_{(1 \div N)_{(1-\delta) 1+\beta \mathbf{B}}}$ and $\lambda_{(N+1 \div 2 N)_{M}}=1-\gamma<1$. Consequently, the largest eigenvalue of the perturbation matrix $\mathbf{M}$ equals the largest eigenvalue of the matrix $(1-\delta) \mathbf{I}+\beta \mathbf{B}$, i.e $\lambda_{1_{M}}=1-\delta+\beta>1$. Therefore, the fixed point $p_{i}^{S}=p^{S}=1, \quad p_{i}^{I}=p^{I}=0$ and $p_{i}^{R}=p^{R}=0, i=\overline{1, N}$, is locally unstable.

Before we state the final theorem, we introduce the following:

Assumption 1. Let $\beta, \delta, \gamma \in(0,1]$, with $\beta \geq \delta$, and let $\rho$ be a complex number, satisfying $\|\rho\| \leq 1$. Let:

$$
\lambda_{1,2}=\frac{c+(1-\gamma) \pm \sqrt{(c-(1-\gamma))^{2}-4 \beta \delta \frac{1-\frac{\delta}{\beta}}{1+\frac{\delta}{\gamma}}}}{2}
$$

with $c=1-\beta \frac{1-\frac{\delta}{\beta}}{1+\frac{\delta}{\gamma}}-\delta+\delta \rho$. Then: $\left\|\lambda_{1,2}\right\| \leq 1$, with equality holding only when $\beta=\delta$ and $\rho=1+\mathrm{i} 0$.

Now, we will state the following theorem: 
Theorem 3. For the SIRS type spreading process, defined with a set of the status probability equations (1), and for $\frac{\delta}{\beta}<1$, the point $p_{i}^{S}=p^{S}=\frac{\delta}{\beta}$, $p_{i}^{I}=p^{I}=\frac{1-\frac{\delta}{\beta}}{1+\frac{\delta}{\gamma}}$, and $p_{i}^{R}=p^{R}=\frac{\delta}{\gamma} \frac{1-\frac{\delta}{\beta}}{1+\frac{\delta}{\gamma}}$, is a locally stable fixed point.

Proof. To prove the theorem, we are first going to introduce the following transformation of variables:

$$
p_{i}^{S}(t)=p_{i}^{S}(t)-\frac{\delta}{\beta}, \quad p_{i}^{\prime I}(t)=p_{i}^{I}(t)-\frac{1-\frac{\delta}{\beta}}{1+\frac{\delta}{\gamma}}, \quad p_{i}^{\prime R}(t)=p_{i}^{R}(t)-\frac{\delta}{\gamma} \frac{1-\frac{\delta}{\beta}}{1+\frac{\delta}{\gamma}} .
$$

By replacing the transformed variables, and by introducing the newly acquired constraint $p_{i}^{\prime S}+p_{i}^{\prime I}+p_{i}^{\prime R}=0$ into the second and the third equation of the system (1), one obtains:

$$
\begin{aligned}
p_{i}^{\prime I}(t+1) & =\left(1-\delta-\beta \frac{1-\frac{\delta}{\beta}}{1+\frac{\delta}{\gamma}}\right) p_{i}^{\prime I}(t)+\left(\frac{\delta}{\beta}-p_{i}^{\prime I}(t)-p_{i}^{\prime R}(t)\right) . \\
& \cdot \sum_{j=1}^{N} \beta b_{i j} p_{j}^{\prime I}(t)-\beta \frac{\delta}{1+\frac{\delta}{\gamma}} p_{i}^{\prime R}(t) \\
p_{i}^{\prime I}(t+1) & =\delta p_{i}^{\prime I}(t)+(1-\gamma) p_{i}^{\prime R}(t) .
\end{aligned}
$$

Next, to prove the local stability of the system (1) in the observed point, we will prove the local stability of the origin of the translated system (7). By perturbing the system (7) at the origin, one obtains:

$$
\begin{aligned}
& \delta p_{i}^{\prime I}(t+1)=\left(1-\delta-\beta \frac{1-\frac{\delta}{\beta}}{1+\frac{\delta}{\gamma}}\right) \delta p_{i}^{\prime I}(t)+\frac{\delta}{\beta} \sum_{j=1}^{N} \beta b_{i j} \delta p_{j}^{\prime I}(t)-\beta \frac{1-\frac{\delta}{\beta}}{1+\frac{\delta}{\gamma}} p_{i}^{\prime R}(t) \\
& \delta p_{i}^{\prime I}(t+1)=\delta\left(\delta p_{i}^{\prime I}(t)\right)+(1-\gamma) \delta p_{i}^{\prime R}(t),
\end{aligned}
$$

or in matrix form: 


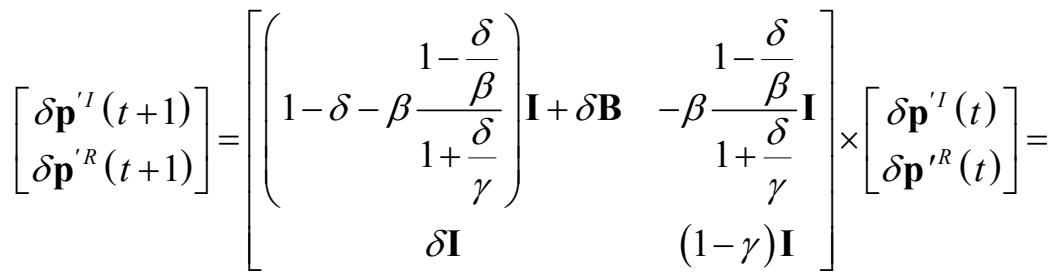

$$
\begin{aligned}
& =\mathbf{M}\left[\begin{array}{l}
\delta \mathbf{p}^{\prime I}(t) \\
\delta \mathbf{p}^{\prime R}(t)
\end{array}\right]
\end{aligned}
$$

Let $\mathbf{u}^{(i)}$ be the right eigenvector, associated to the $i-t h$ eigenvalue $\lambda_{i_{s}}$ of the matrix $\mathbf{S}=\left(1-\delta-\beta \frac{1-\frac{\delta}{\beta}}{1+\frac{\delta}{\gamma}}\right) \mathbf{I}+\delta \mathbf{B}$. It is obvious that $\mathbf{u}^{(i)}$ is eigenvector for the matrices $-\beta \frac{1-\frac{\delta}{\beta}}{1+\frac{\delta}{\gamma}} \mathbf{I}, \delta \mathbf{I}$ and $(1-\gamma) \mathbf{I}$, as well. Therefore, for the eigenvector $\mathbf{w}^{(j)}$ corresponding to the to $j-$ th eigenvalue of the matrix $\mathbf{M}, \lambda_{j_{\mathbf{M}}}$, one could consider the following form: $\mathbf{w}^{(j)}=\left[k_{1} \mathbf{u}^{(i)} k_{2} \mathbf{u}^{(i)}\right]^{T}$, with $k_{1} k_{2}$ being constants, that in general may be complex numbers. Then, from the eigenvalue equation $\mathbf{M} \mathbf{w}^{(j)}=\lambda_{j_{\mathbf{M}}} \mathbf{w}^{(j)}$, and the equation (8), one obtains:

$$
\begin{gathered}
k_{1} \lambda_{i_{\mathrm{S}}} \mathbf{u}^{(i)}-k_{2} \beta \frac{1-\frac{\delta}{\beta}}{1+\frac{\delta}{\gamma}} \mathbf{u}^{(i)}=k_{1} \lambda_{j_{\mathbf{M}}} \mathbf{u}^{(i)} \\
k_{1} \delta \mathbf{u}^{(i)}+k_{2}(1-\gamma) \mathbf{u}^{(i)}=k_{2} \lambda_{j_{\mathbf{M}}} \mathbf{u}^{(i)}
\end{gathered}
$$

or

$$
\left(k_{1}\left(\lambda_{i_{\mathrm{S}}}-\lambda_{j_{\mathrm{M}}}\right)-k_{2} \beta \frac{1-\frac{\delta}{\beta}}{1+\frac{\delta}{\gamma}}\right) \mathbf{u}^{(i)}=\mathbf{0}_{N \times 1}
$$




$$
\left(k_{1} \delta+k_{2}\left(1-\gamma-\lambda_{j_{\mathbf{M}}}\right)\right) \mathbf{u}^{(i)}=\mathbf{0}_{N \times 1} .
$$

In order the system (9) has solution in respect to $k_{1}$ and $k_{2}$, confirming that the assumption about the form of the eigenvectors $\mathbf{w}^{(j)}$ is valid, the following relation should be satisfied:

$$
\left|\begin{array}{cr}
\lambda_{i_{\mathrm{S}}}-\lambda_{j_{\mathrm{M}}} & -\beta \frac{1-\frac{\delta}{\beta}}{1+\frac{\delta}{\gamma}} \\
\delta & 1-\gamma-\lambda_{j_{\mathrm{M}}}
\end{array}\right|=0,
$$

from which one may find the values of $\lambda_{j_{\mathrm{M}}}$ 's:

$$
\lambda_{j 1, j 2_{\mathrm{M}}}=\frac{\lambda_{i_{\mathrm{s}}}+(1-\gamma) \pm \sqrt{\left(\lambda_{i_{\mathrm{s}}}-(1-\gamma)\right)^{2}-4 \beta \delta \frac{1-\frac{\delta}{\beta}}{1+\frac{\delta}{\gamma}}}}{2}
$$

In the last equation $\lambda_{i_{\mathrm{s}}}=1-\delta-\beta \frac{1-\frac{\delta}{\beta}}{1+\frac{\delta}{\gamma}}+\delta \lambda_{i_{\mathrm{B}}}$. From the Perron-Frobenius theorem, the largest eigenvalue of the matrix $\mathbf{B}, \lambda_{\mathbf{B}}=1$ is distinctive, with all other eigenvalues $\left\|\lambda_{i_{\mathrm{B}}}\right\| \leq 1$. If Assumption 1 holds, then $\left\|\lambda_{j 1, j 2_{\mathrm{M}}}\right\|<1$, for any $\beta, \delta, \gamma \in(0,1)$ satisfying the terms stated in the Theorem. The proof is completed.

Remark 1. The proof of the last theorem relies on the correctness of the Assumption 1. We have numerically investigated the claim, taking different values of $\beta, \delta, \gamma$ with step 0.01, considering that $\beta>\delta$ and $\rho$ with step 0.01 for both the module and the argument. The numerics indicated that for all considered combinations (total of 5, 099, 490, 000), Assumption 1 holds. 
Remark 2. The last two theorems confirm that the point $p_{i}^{S}=p^{S}=\frac{\delta}{\beta}$, $p_{i}^{I}=p^{I}=\frac{1-\frac{\delta}{\beta}}{1+\frac{\delta}{\gamma}}$ and $p_{i}^{R}=p^{R}=\frac{\delta}{\gamma} \frac{1-\frac{\delta}{\beta}}{1+\frac{\delta}{\gamma}}$ may be a valid solution of the system (1), for $\frac{\delta}{\beta}<1$. However, since global asymptotic stability was not proven, this does not exclude the possibility that another solution in a form of a fixed point, periodic orbit, limit tori or chaotic attractor exists for the studied system.

From the previous discussion, we may define the average number of susceptible nodes in the network, $N_{S}=\lim _{t \rightarrow \infty} \sum_{i=1, N} p_{i}^{S}(t)$, the average number of infected nodes in the network, $N_{I}=\lim _{t \rightarrow \infty} \sum_{i=1, N} p_{i}^{I}(t)$ and the average number of removed nodes in the network, $N_{R}=\lim _{t \rightarrow \infty} \sum_{i=1, N} p_{i}^{R}(t)$, for different parametric regions:

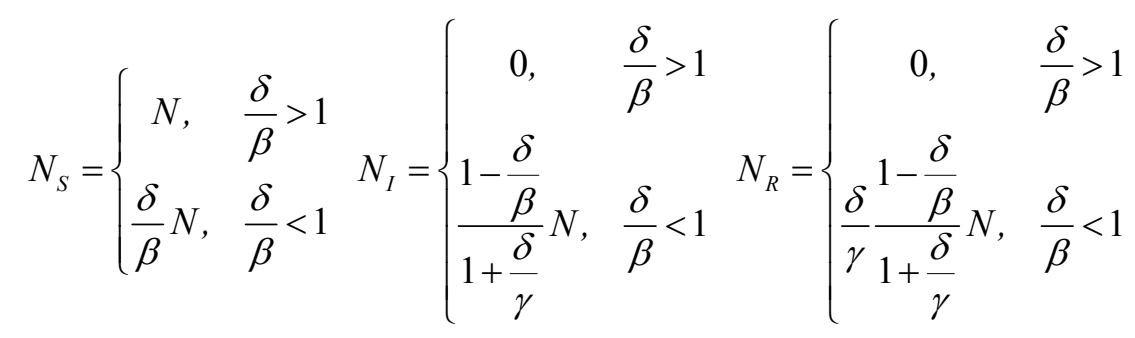

As in $[25,26]$, the obtained results are topology independent.

\section{NUMERICAL RESULTS}

In order to check the validity of the results obtained in the previous sections, as well as to investigate the regions in which they adequately mimic the reality, in this section the results of stochastic simulations (the reality) are presented and compared with those analytically obtained. We use the same testing protocols as in $[25,26]$. The simulations are performed on Barabási-Albert 
graphs (in the following text we use the notation $\mathrm{BA}\left(m, m_{0}, N\right)$ ), where the minimal node degree is being altered by changing the parameter $m$. We focus on BA graphs, expecting that the statistical independence of joint events hypothesis will be less valid on networks where the nodes with minimal node degree are in large quantity. In that sense, BA graphs are the 'worst case scenario' for the tested process, when considering computer generated networks that mimic real world graphs. Identically as in $[25,26]$, the stochastic simulations are performed so that first the system network-infection, starting from arbitrary initial conditions, is iterated for 2000 time steps in order the system reaches the stationary regime. Then the system is further iterated for another 8000 time steps, after which the results are averaged. The analysis is repeated several times for different values of the parameters $\beta, \delta$ and $\gamma$. In the analysis, connection probabilities $p(i \leftarrow j)$ have been chosen randomly for each existing link.

The results of the analysis are presented in Figs. 1 and 2.

Results from the numerical simulations suggest that similar conclusions as those obtained for the SIS spreading process in $[25,26]$, may be drawn for the SIRS process occurring under the circumstances studied in this paper, as well. As shown in Fig. 1 the validity of the analytical results increases for large $\beta / \delta$ ratios, and when larger values of $\beta$ are considered. In addition, from the figure, one may conclude that parameter $\gamma$ is positively correlated with the accuracy of the analytical results: as $\gamma$ increases the analytical results better mimic the reality (results of the stochastic simulation). As presented in Fig. 2, by taking large values for both $\beta$ and $\gamma(\beta=\gamma=1)$, a good match between the analytical results and the reality may be achieved for a wide range of values for the parameter $\delta$. As Fig. 2 further indicates, the accuracy of the obtained results, identically as for the SIS type process [25, 26], increases with the increase of the minimal node degree in the network.

The obtained results suggest that the SIRS type of spreading process, identically as described in [25, 26], under certain conditions, may be used as a basis for controlled spreading of useful content in technological networks. However, one must conclude that the SIS process, presented in our previous work is more suitable for this purpose. The reasons for that lays in the fact that the accuracy of the analytical results is more easily achieved for the SIS process, in respect to the minimal node degree in the network. Next, the existence of an additional status ('removed') has an adverse affect on the validity of the hypothesis of statistical independence of join events. 


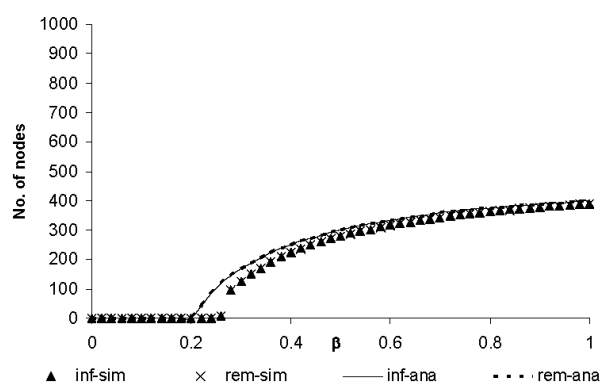

a)

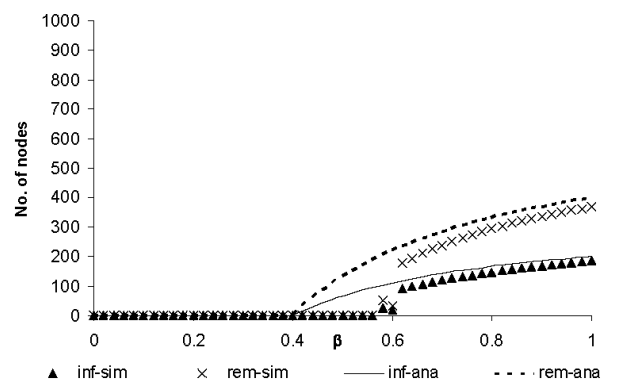

c)

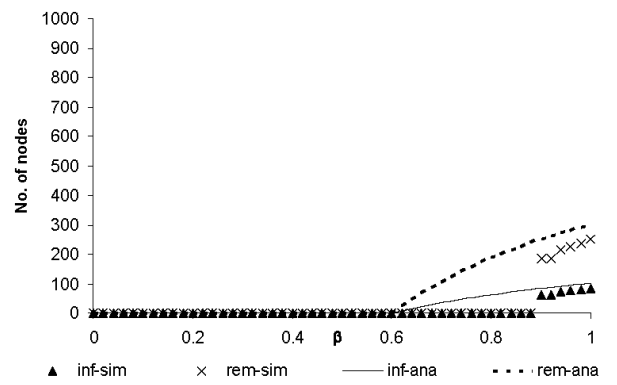

e)

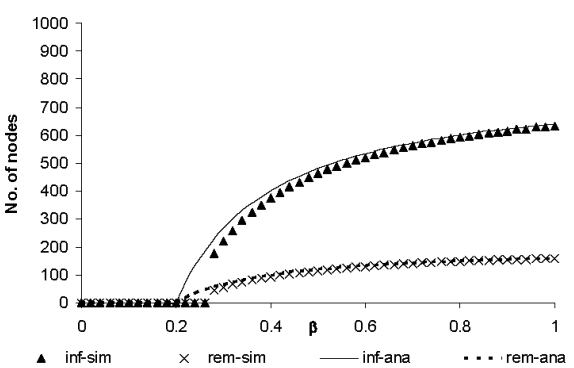

b)

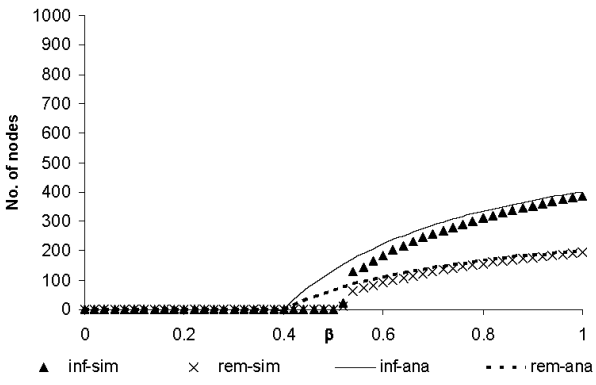

d)

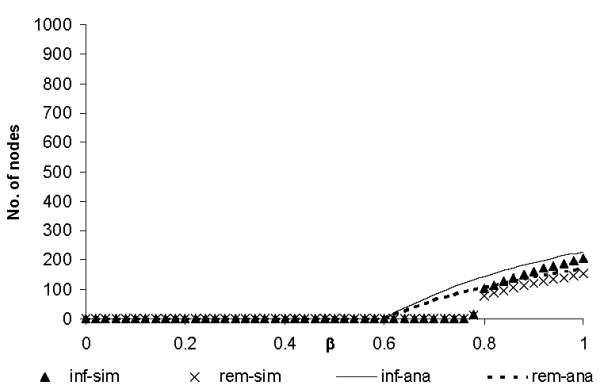

f)

Figure 1. Analysis the SIRS type process occurring on the BA(7,8,1000) network as a function of $\beta$ for: a) $\delta=0.2$ and $\gamma=0.2$; b) $\delta=0.2$ and $\gamma=0.8$; c) $\delta=0.4$ and $\gamma=0.2$; d) $\delta=0.4$ and $\gamma=0.8$; e) $\delta=0.6$ and $\gamma=0.2$, f) $\delta=0.6$ and $\gamma=0.8$. The number of infected nodes obtained from the stochastic simulation (inf-sim), analytically obtained number of infected nodes (inf-ana), number of removed nodes obtained from the stochastic simulation (rem-sim) and analytically obtained number of removed nodes (rem-ana) are presented 


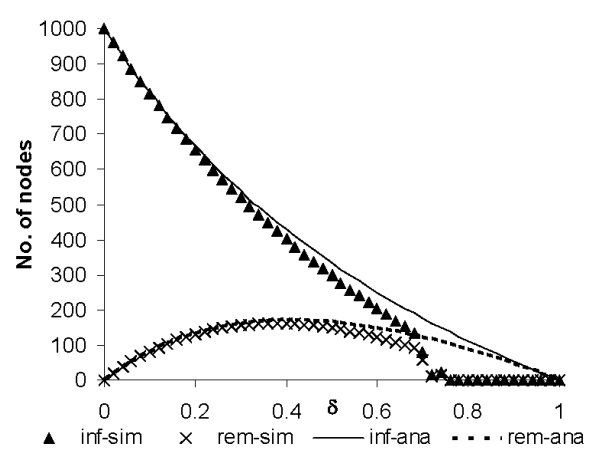

a)

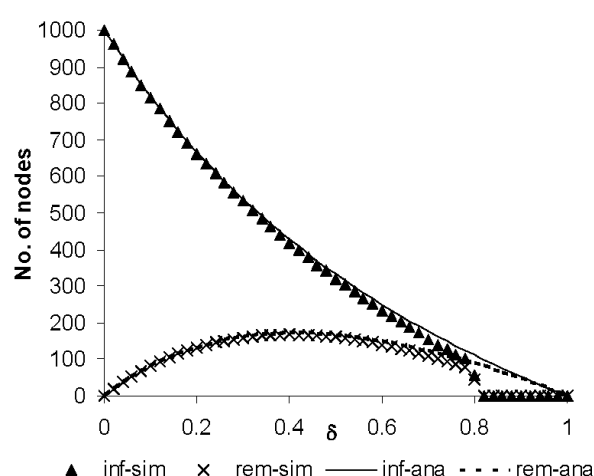

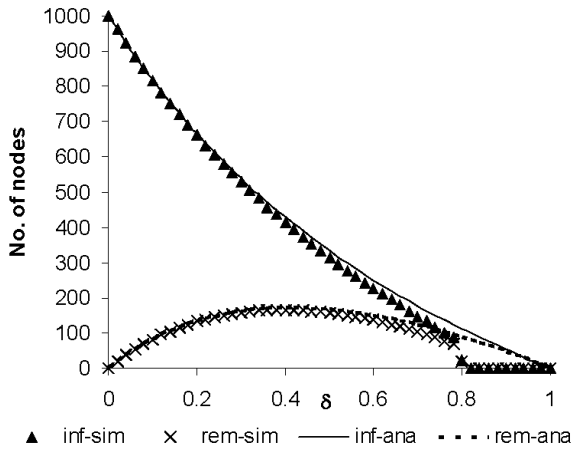

b)

Figure 2. Analysis the SIRS type process for different values of the parameter $\delta$, when $\beta=\gamma=1$, a) $\mathrm{BA}(4,5,100)$, b) $\mathrm{BA}(7,8,1000)$, c) $\mathrm{BA}(10,11,1000)$ network. The number of infected nodes obtained from the stochastic simulation (inf-sim), analytically obtained number of infected nodes (inf-ana), number of removed nodes obtained from the stochastic simulation (rem-sim) and analytically obtained number of removed nodes (rem-ana) are presented

\section{CONCLUSION}

We have shown that the SIRS type processes occurring on networks characterized by acquisition exclusivity and in circumstances where statistical independence of join events may be assumed is analytically solvable and the obtained results are independent of the network topology. This could be used as a basis for development of practical applications for controlled sharing of useful content in technical networks. However, the results from the stochastic simulations suggest that the results previously obtained for the SIS process are more promising for this purpose. 


\section{REFERENCES}

[1] R. Pastor-Satorras and A. Vespignani: "Epidemic spreading in scale-free networks", Physical Review Letters, vol. 86, no. 14, pp. 3200-3203 (2001).

[2] R. Pastor-Satorras and A. Vespignani: "Epidemic dynamics and endemic states in complex networks", Physical Review E, vol. 63, no. 6, 066117 (2001).

[3] C. Moore and M. E. J. Newman: "Epidemics and percolation in small-world networks", Physical Review E, vol. 61, no. 5, pp. 5678-5682 (2000).

[4] M. E. J Newman: "Spread of epidemic desease on networks", Physical Review E, vol. 66, no. 1, art. 016128 (2002).

[5] R. Parshani, S. Carmi and S. Havlin: "Epidemic Threshold for the SusceptibleInfectious-Susceptible Model on Random Networks", Physical Review Letters, vol. 104, no. 25, art. 258701 (2010).

[6] Y. Wang, D. Chakrabarti, C. Wang, and C. Faloutsos: "Epidemic Spreading in Real Networks: An Eigenvalue Viewpoint", Proceedings of the 22nd International Symposium on Reliable Distributed Systems (IEEE SRDS'03), , Firence, Italy, pp. 25-34 (2003).

[7] D. Chakrabarti, Y. Wang, C. Wang, J. Leskovec and C. Faloutsos: "Epidemic Thresholds in Real Networks". ACM Transactions on Information and System Security, vol. 10, no. 4, art. 13, Jan. 2008.

[8] P. Van Mieghem, J. Omic, Robert Kooij: "Virus Spread in Networks", IEEE/ACM Trans. Netw., vol. 17, no. 1, pp. 1-14, Feb. 2009.

[9] A. Ganesh, L. Massoulie, and D. Towsley: "The Effect of Network Topology on the Spread of Epidemics", in Proc. IEEE Infocom, vol. 2, Miami, Fl., Mar. 2005, pp. 1455-1466.

[10] R. Pastor-Satorras and A. Vespignani: "Immunization of complex networks", Physical Review E, vol. 65, no. 3, art. 036104 (2002).

[11] Y. Wan, S. Roy and A.Saberi: "Network design problems for controlling virus spread", Proceedings of 46th IEEE Conference on Decision and Control, New Orleans, LO, Dec. 2007, pp. 3925-3932.

[12] Y. Wan, S. Roy and A. Saberi: "Designing spatially-heterogeneous strategies for control of virus spread", IET Systems Biology. vol. 2, no. 4, pp. 184-201, Jul. 2008.

[13] J. Omić, J. Martìn-Hernández, and P. Van Mieghem, "Network protection against worms and cascading failures using modularity partitioning", In: Proc. 22nd International Teletraffic Congress (ITC 22), Amsterdam, Netherlands, Sep. 2010, pp. $1-8$.

[14] H. F. Zhang, K. Z. Li, X. C. Fu, and B. H. Wanh, "An Efficient Control Strategy of Epidemic Spreading on Scale-Free Networks", Chinese Phys. Lett., vol. 26, no. 6, pp. 068901/1 - 068901/4, Jun. 2009.

[15] I. Tomovski and L. Kocarev, "Simple Algorithm for Virus Spreading Control on Complex Networks", IEEE Trans. Circuits Syst. I: Regular Papers, vol. 59, no. 4, pp. 763-771, Apr. 2012. 
[16] B. A. Carreras, D. E. Newman, I. Dobson, and A. B. Poole, "Evidence for SelfOrganized Criticality in a Time Series of Electric Power System Blackouts", IEEE Transactions on Circuits and Systems I: Regular papers, vol. 51, no. 9, pp. 17331740, Sep. 2004.

[17] I. Dobson, B. A. Carreras, and D. E. Newman, "Probabilistic load-dependent cascading failure with limited component interactions", in Proc. IEEE Int. Symp. Circuits Syst., Vol. 5, Vancouver, Canada, May 2004, pp. V-912-V-915.

[18] D. E. Newman, B. A. Carreras, V. E. Lynch, and I. Dobson, "Exploring complex systems aspects of blackout risk and mitigation", IEEE Trans. Reliab., vol. 60, no. 1, pp. 134-143, Mar. 2011.

[19] B. A. Carreras, V.E. Lynch, D. E. Newman, and I. Dobson, "Critical points and transitions in an electric power transmission model for cascading failure blackouts", Chaos, Vol. 12, No. 4, pp. 985-994, Dec. 2002.

[20] B. A. Carreras, V.E. Lynch, I. Dobson, and D. E. Newman, "Complex dynamics of blackouts in power transmission systems", Chaos, vol. 14, no. 3, pp. 643-652, Sep. 2004.

[21] J. Chen, J. S. Thorp, and I. Dobson, "Cascading dynamics and mitigation assessment in power system disturbances via a hidden failure model", International Journal of Electrical Power and Energy Systems, vol. 27, no. 4, pp. 318-326, May 2005.

[22] P. Crucitti, V. Latora, M. Marchiori and A. Rapisarda, "Efficiency of scale-free networks: error and attack tolerance", Physics A, vol. 320, pp. 622-642, Mar. 2003.

[23] P. Crucitti, V. Latora, M. Marchiori and A. Rapisarda, "Error and attack tolerance of complex networks", Physics A, vol 340, no. 1-3, pp. 388-394, Sep. 2004.

[24] I. Petreska, I. Tomovski, E. Gutierrez, L. Kocarev, F. Bono and K. Poljansek, "Application of modal analysis in assessing attack vulnerability of complex networks", Communications in Nonlinear Science and Numerical Simulation, Vol. 15, no. 4, pp. 1008-1018, Apr. 2010.

[25] I. Tomovski, I.Trpevski, L. Kocarev, "Topology Independent SIS Process: Theory and Application," In: Proceedings of the International Conference on Theory and Applications in Nonlinear Dynamics - ICAND2012, Seattle, OR, Aug. 2012, To be published.

[26] I. Tomovski, I. Trpevski and L. Kocarev, "Topology Independent SIS Process: an engineering viewpoint", Submited to Communications in Nonlinear Science and Numerical Simulation.

[27] S. Gomez, A. Arenas, J. Borge-Holthoefer, S. Meloni, and Y. Moreno, "Discretetime Markov chain approach to contact-based disease spreading in complex networks", Eur. Phys. Lett., vol. 89, no.3, pp. 38009/p1-38009/p6, Feb.2010.

[28] S. Gomez, A. Arenas, J. Borge-Holthoefer, S. Meloni and Y. Moreno, "Probabilistic framework for epidemic spreading in complex networks,Int. J. Complex Systems in Science, vol. 1, pp. 47-51, 2011. 
[29] K. Suchecki, V. M. Eguiluz and M. San Miguel, "Voter model dynamics in complex networks: Role of dimensionality, disorder, and degree distribution", Phys. Rev. E, vol. 72, no. 3, pp. 036132/1-036132/8, Sep. 2005.

Р е $з$ и м е

ТОПОЛОШКИ НЕЗАВИСЕН SIRS-ПРОЦЕС

Следејќи го нашето претходно истражување за тополошки независниот SIS-модел на ширење [25, 26], во оваа статија анализираме SIRS-тип на процес кој се одвива на мрежи карактеризирани со посебен тип контактна динамика, за која го користиме терминот „аквизициска ексклузивност". Се покажува дека за разгледуваниот систем, идентично како и за процесот од SIS-тип, во услови кога може да се претпостави статистичка независност на здружените настани, може да се најде аналитичко решение за веројатностите секој од јазлите да се наоѓa во определен статус во стационарен режим. При тоа, аналитичките резултати укажуваат на тоа дека процесот од SIRS-тип, при околностите во кои се анализира е тополошки независен.

Клучни зборови: комплексни мрежи; SIRS-процес; нелинеарни системи

Address:

\section{Igor Tomovski}

Research Center for Energy Informatics and Materials,

Macedonian Academy of Sciences and Arts

Bul. Krste Misirkov 2, 1000 Skopje, Macedonia

igor@manu.edu.mk

\section{Igor Trpevski}

Research Center for Energy Informatics and Materials,

Macedonian Academy of Sciences and Arts

Bul. Krste Misirkov 2, 1000 Skopje, Macedonia

igortrpevski@gmail.com

\section{Ljupčo Kocarev}

Research Center for Energy Informatics and Materials,

Macedonian Academy of Sciences and Arts

Bul. Krste Misirkov 2, 1000 Skopje, Macedonia

lkocarev@ucsd.edu 